\title{
New Developments in the Biodemography of Aging and Longevity
}

\author{
Leonid A. Gavrilov Natalia S. Gavrilova \\ Center on Aging, NORC at the University of Chicago, Chicago, III., USA; WHO Collaborating Centre, Department of \\ Statistical Analysis of Population Health, Federal Research Institute for Health Organization and Informatics, \\ Ministry of Health of the Russian Federation, Moscow, Russia
}

\begin{abstract}
Key Words
Biodemography $\cdot$ Longevity · Mortality trajectories ·

Maternal age $\cdot$ Centenarians
\end{abstract}

\begin{abstract}
Biodemography is a promising scientific approach based on using demographic data and methods for getting insights into biological mechanisms of observed processes. Recently, new important developments have happened in biodemographic studies of aging and longevity that call into question conventional aging theories and open up novel research directions. Recent studies found that the exponential increase of the mortality risk with age (the famous Gompertz law) continues even at extreme old ages in humans, rats, and mice, thus challenging traditional views about oldage mortality deceleration, mortality leveling-off, and latelife mortality plateaus. This new finding represents a challenge to many aging theories, including the evolutionary theory that explains senescence by a declining force of natural selection with age. Innovative ideas are needed to explain why exactly the same exponential pattern of mortality growth is observed not only at reproductive ages, but also at very-old postreproductive ages (up to 106 years), long after the force of natural selection becomes negligible (when there is no room for its further decline). Another important recent development is the discovery of long-term 'memory' for early-life experiences in longevity determination. Siblings born to young mothers have significantly
\end{abstract}

higher chances to live up to 100 years, and this new finding, confirmed by two independent research groups, calls for its explanation. As recent studies found, even the place and season of birth matter for human longevity. Beneficial longevity effects of young maternal age are observed only when children of the same parents are compared, while the maternal age effect often could not be detected in acrossfamilies studies, presumably being masked by betweenfamily variation. It was also found that male gender of centenarian has a significant positive effect on the survival of adult male biological relatives (brothers and fathers) but not of female relatives. Finally, large gender differences are found in longevity determinants for males and females, suggesting a higher importance of occupation history for male centenarians as well as a higher importance of home environment history for female centenarians.

(C) 2014 S. Karger AG, Basel

\section{Introduction}

Recent important developments in biodemographic studies of aging and longevity call into question conventional aging theories and open up novel research directions. In this review, we cover new developments in two broad areas of biodemographic research: (1) studies of mortality laws in humans and laboratory animals at extreme old ages, and (2) studies on predictors of exceptional human longevity.

\section{KARGER 125}

(c) 2014 S. Karger AG, Base

0304-324X/14/0614-0364\$39.50/0

E-Mail karger@karger.com

www.karger.com/ger
Leonid A. Gavrilov

Center on Aging

NORC at the University of Chicago

1155 E 60th Street, Chicago, IL 60637 (USA)

E-Mail gavrilov@longevity-science.org 


\section{Biodemography of Old-Age Mortality}

Attempts to develop a fundamental quantitative theory of aging, mortality, and life span have deep historical roots. In 1825, the British actuary Benjamin Gompertz discovered a law of mortality [for reviews, see 1-4]. Specifically, he found that the force of mortality increases in geometrical progression with the age of adult humans. According to the Gompertz law, human mortality rates double about every 8 years of adult age. An exponential (Gompertzian) increase in death rates with age is observed for many biological species, including fruit flies (Drosophila melanogaster), nematodes, mosquitoes, human lice (Pediculus humanus), flour beetles (Tribolium confusum), mice, rats, dogs, horses, mountain sheep, and baboons [for reviews, see 1,2].

It was also believed that the exponential growth of mortality with age (Gompertz law) is followed by a period of deceleration, with slower rates of mortality increase at extreme old ages $[1,5]$. This mortality deceleration eventually produces the 'late-life mortality leveling-off' and 'late-life mortality plateaus' at extreme old ages. Greenwood and Irwin [5] provided a detailed description of this phenomenon in humans and even made the first estimates for the asymptotic value of the upper limit to human mortality. The same phenomenon of 'almost nonaging' survival dynamics at extreme old ages was described for other biological species, and in some species such as medfly and housefly, the mortality plateau can occupy a sizable part of their life $[2,6]$.

According to some researchers [7], the late-life mortality plateau represents a distinct phase of life when aging slows down or stops. Evolutionary biologists believe that aging is a result of the declining forces of natural selection with age. When these forces eventually bottom at extreme old ages, then the cessation of aging is expected according to this paradigm [7]. The population heterogeneity hypothesis is another, even more popular, explanation of mortality deceleration, which was proposed by British actuary Robert Eric Beard in 1959 [8]. As George Sacher explained '.. sub-populations with the higher injury levels die out more rapidly, resulting in progressive selection for vigour in the surviving populations' [9, p. 435]. Another explanation of this phenomenon comes from the reliability theory of aging, which explains mortality leveling-off by an exhaustion of the organism's redundancy (reserves) at extremely old ages, so that every additional random hit of damage results in death $[1,2]$. There is also an opinion that lower (than predicted) risks of death for older people may be due to their less risky behavior [5].

New Developments in the Biodemography of Aging and Longevity
The existence of mortality plateaus is well described for a number of lower organisms, including medflies, house flies (Musca domestica), fruit flies (Anastrepha ludens, A. obliqua, and A. serpentine), parasitoid wasps (Diachasmimorpha longicaudata), and bruchid beetles (Callosobruchus maculatus) [for a review, see 2]. In the case of mammals, however, data are much more controversial. Sacher [9] reported short-term periods of mortality deceleration at advanced ages in mouse populations and even used the Perks formula for mortality modeling in his analyses. However, Austad [10] later argued that rodents do not demonstrate mortality deceleration even for very large samples. A study in baboons found no mortality deceleration at older ages [11]. Similarly, a longitudinal study by Bronikowski et al. [12] of the mortality among 7 wild primate species failed to find a mortality deceleration at older ages. The authors concluded that 'none of the age-specific mortality relationships in our non-human primate analyses demonstrated the type of leveling off that has been shown in human and fly data sets' [12, p. 1328]. Thus, we may suggest that mortality deceleration is observed for many invertebrate species, but the evidence for mammals is controversial.

Several studies of old-age mortality in humans came to the conclusion that mortality deceleration does exist and starts after the age of 80 years $[13,14]$. It should be noted, however, that analysis of old-age mortality in humans encounters certain methodological problems related to data aggregation and age misreporting among very old individuals. More homogeneous single-year birth cohorts in many countries with good vital statistics have very small numbers of survivors up to the age of 100 years that makes estimates of mortality at advanced ages unreliable. On the other hand, aggregation of data for several birth cohorts in order to increase the sample size creates a mixture of different populations. The problem of age misreporting by older persons is another important problem affecting estimates of mortality at advanced ages. It was found that mortality deceleration is more expressed in the case of data with poor quality compared to data with better quality [15].

Recently, new developments have happened in this research area thanks to the use of more detailed and more accurate data. In particular, the US Social Security Administration's Death Master File (DMF) was used for an accurate estimation of hazard rates at extremely old ages in extinct birth cohorts. The availability of information about the month of birth and the month of death in this data source provides a unique opportunity to obtain more accurate hazard rate estimates for every month of 
age. A study of 20 single-year extinct birth cohorts with good data quality found that mortality deceleration at advanced ages is negligible up to the advanced age of 106 years [15]. This finding was further supported by additional studies of mortality in 22 single-year US birth cohorts based on data from the Human Mortality Database and data on the mortality of 1,681 siblings of centenarians $[16,17]$. The same conclusion was drawn after an analysis of mortality trajectories in 8 cohorts of laboratory mice and 10 cohorts of laboratory rats [17]. Thus, for all 3 mammalian species, the Gompertz model fits the mortality data significantly better than the Kannisto model predicting mortality deceleration (according to the Akaike information criterion as the goodness-of-fit measure) [17].

There are several reasons why earlier studies $[13,14]$, including our own research $[1,2]$, reported mortality deceleration and mortality leveling-off at advanced ages. First, mortality deceleration may be caused by age misreporting in death data for older individuals $[15,18]$. Studies conducted more than 10 years ago used data for older birth cohorts when age reporting was not particularly accurate. In the United States, this may have impaired the accuracy of mortality rate estimates in the past.

Second, mortality deceleration may be a consequence of data aggregation. Most developed countries have much smaller populations compared to the United States, and hence studies of mortality at advanced ages for these countries have to combine many single-year birth cohorts, thereby increasing the heterogeneity of the sample.

Also, some researchers used inappropriate estimates of the instantaneous mortality rate (hazard rate). At the most advanced ages, the rates of death are so high it is impossible to assume that the number of dying is distributed uniformly within the studied 1-year age intervals. As a result, the estimates of mortality rates (or central death rates) are biased downward at advanced ages. Many studies analyzed the age-specific probability of death rather than the hazard rate, which is biased downward at old ages $[19,20]$. It is not surprising that the probability of death has a tendency of deceleration at advanced ages when mortality is high, taking into account that this mortality indicator has a theoretical upper limit equal to 1 . For example, a study of mortality among supercentenarians demonstrated that the probability of death for this group does not increase with age [20].

These results suggest that mortality deceleration at advanced ages is not a universal phenomenon, and that survival of mammalian species follows the Gompertz law up to very old ages. This new finding represents a challenge to many aging theories, including the evolutionary theory that explains senescence by a declining force of natural selection with age. Novel ideas are needed to explain why exactly the same exponential pattern of mortality growth is observed not only at reproductive ages, but also at very old postreproductive ages (up to 106 years), long after the force of natural selection becomes negligible (when there is no room for its further decline).

The finding that the Gompertz law might be applicable in a wide range of adult ages leads to another burning research question: how is it possible for different diseases and causes of death to 'negotiate' with each other in order to produce a simple exponential function for all-cause mortality (given that the contribution of different causes of death to all-cause mortality changes dramatically with age)?

\section{Biodemography of Exceptional Longevity}

The search for longevity predictors is another promising and rapidly developing direction of biodemographic studies. Centenarians (persons living up to the age of 100 years and over) represent a population that could be useful for identifying factors leading to long life and avoidance of fatal diseases. Even if some early- or midlife factors have a moderate positive effect on the risk of death, persons with this quality will be accumulated among long-lived individuals. Thus, the study of centenarians may be a sensitive way to find genetic, familial, environmental, and life-course factors associated with lower mortality and better survival.

It has been shown that many centenarians have been healthy or well-functioning throughout most of their lives; thus, they represent a useful model for delayed aging [21]. Contemporary populations are subjected to rapid population aging; therefore, identifying the pathways to healthy longevity is of particular importance. These are important issues not only for demographic forecasts of mortality and population aging; rather, they also have policy implications for health-care and pension expenditures and are important for improving our understanding of the fundamental mechanisms of human aging and longevity.

Many studies of centenarians have focused on the search for longevity genes, while other important predictors of exceptional longevity have often been overlooked. Of particular interest are factors of early-life developmental programming as an important determinant of health and survival in later life as well as the corollary hypothesis 
Table 1. Predictors of survival up to the age of 100 years: effects of parental longevity and early- and midlife conditions

\begin{tabular}{|c|c|c|c|c|c|c|}
\hline Variable & odds ratio & $95 \% \mathrm{CI}$ & $\mathrm{p}$ value & odds ratio & $95 \% \mathrm{CI}$ & $\mathrm{p}$ value \\
\hline Mother lived $80+$ & 1.94 & $1.41-2.65$ & $<0.001$ & 2.33 & $1.71-3.17$ & $<0.001$ \\
\hline Farmer in $1930^{*}$ & 1.79 & $1.21-2.31$ & $<0.001$ & 1.21 & $0.87-1.66$ & 0.253 \\
\hline Born in the northeast part of the USA & 1.86 & $1.14-3.03$ & 0.013 & 0.98 & $0.60-1.59$ & 0.931 \\
\hline
\end{tabular}

The results are from multivariate logistic regression.

* Husband or head of household was a farmer in 1930 (for women).

stating that infectious diseases in childhood have long-lasting effects on subsequent survival at advanced ages [22].

In our most recent studies, we used computerized family histories as a primary source of information about long-lived individuals (centenarians). Family histories (genealogies) have proven to be a useful source of information for studies in historical demography and biodemography. Making use of a substantial body of information available in online historical sources allowed us to test a number of hypotheses regarding links between early-life conditions, middle-age experiences, and exceptional longevity.

\section{Why Centenarians Are Different from Their}

Shorter-Lived Peers

The prevalence of centenarians in modern populations is very low (about 1 per 10,000 [23]), and, therefore, traditional methods of population sampling are difficult and not feasible for obtaining large samples of centenarians. The case-control design has proven to be the most appropriate and cost-effective approach for studies of rare conditions and is extremely useful for centenarian studies. The control selection strategy in this study was to avoid bias arising from noncomparability between cases and controls. In order to minimize this bias, the cases and controls were selected from the same population universe (i.e., they were selected from the same pool of computerized family histories).

The availability of data on US centenarians and their shorter-lived peers (died at the age of 65 years) born in 1890-1891 allowed us to test several hypotheses on earlylife conditions and longevity in a straightforward manner. This approach confirmed that parental longevity is a strong independent predictor of survival up to the age of

New Developments in the

Biodemography of Aging and Longevity
100 years, so this variable cannot be ignored in gerontological studies (table 1). At the same time, early exposure to infections as estimated indirectly from child mortality in the families of cases and controls had no effect on longevity. Overall, childhood conditions reported in the 1900 census were not predictive for exceptional longevity for either men or women [24]. On the other hand, some early-life characteristics (birth in the northeastern part of the United States and birth in the second half of the year) turned out to be significant predictors of exceptional longevity (for men but not women). This study also found a strong positive effect of farmer occupation at middle age on attaining exceptional longevity for men. For women, however, a farmer occupation of their husbands had no effect on longevity. This finding is in agreement with the results of other studies, including our earlier study of centenarians based on a population-based sample of survivors up to the age of 100 years from the 1887 birth cohort [25]. Only a few factors were related to the exceptional longevity of women: parental longevity and, surprisingly, the availability of radio in household in 1930 [24]. The effects of radio as a proxy for household wealth might potentially explain the latter finding. However, more direct characteristics of household wealth (whether it was owned or rented property) demonstrated no association with exceptional longevity. Earlier studies found that radio listening increased quality of life and decreased depression [26]. This study demonstrated that only few selected factors turned out to be significant predictors of survival after the age of 65 years, while many other earlyand midlife living conditions do not significantly affect mortality at this age period. It also revealed significant gender differences in the spectrum of predictors of exceptional longevity. 
Table 2. Mean life span (95\% CI) conditional on survival up to the age of 50 years (LS50) for relatives of centenarians compared to the 1900 US birth cohort

\begin{tabular}{llllll}
\hline & \multicolumn{2}{c}{ Male relatives } & & \multicolumn{2}{l}{ Female relatives } \\
\cline { 2 - 3 } \cline { 5 - 6 } & sample size, $\mathrm{n}$ & LS50, years & & sample size, $\mathrm{n}$ & LS50, years \\
\hline Relatives of centenarians & & & & \\
$\quad$ Parents & 1,590 & $76.2(75.7-76.8)$ & & 1,557 & $77.2(76.7-77.8)$ \\
All siblings & 5,324 & $77.6(77.3-77.9)$ & & 4,877 & $82.4(82.0-82.7)$ \\
Married siblings & 3,221 & $77.7(77.3-78.1)$ & & 3,028 & $82.2(81.8-82.6)$ \\
$\quad$ Spouses & 876 & $75.4(74.6-76.1)$ & & 283 & $81.4(80.1-82.7)$ \\
$\quad$ Siblings-in-law & 2,349 & $75.0(74.6-75.5)$ & & 2,407 & $79.5(79.0-79.9)$ \\
1900 US birth cohort & & 73.3 & & 79.4 \\
\hline
\end{tabular}

Effect of the Gender of Centenarians on the Survival of Their Relatives

Numerous studies have demonstrated that biological relatives of centenarians have a substantial survival advantage compared to relatives of shorter-lived individuals. At the same time, little is known about the role of centenarian gender in these effects. We explored the impact of centenarian gender on the survival of their biological (parents and siblings) and nonbiological (spouses and siblings-in-law) relatives using a database of 1,945 validated US centenarians born in 1880-1895 as well as their siblings, spouses, and siblings-in-law.

The comparison of the mean life span of adult relatives (those who survived up to the age of 50 years) reveals a survival advantage for brothers and sisters of centenarians compared to the same-sex siblings-in-law: on average, brothers lived 2.6 and sisters 2.9 years longer than siblings-in-law of the same sex (table 2), with the differences in life span being statistically significant $(\mathrm{p}<$ 0.001 ). Although fathers of centenarians are born about 30 years earlier than brothers-in-law of centenarians, they still have a higher life span conditional on the survival up to the age of 50 years than later-born nonbiological relatives such as siblings-in-law $(\mathrm{p}<0.001)$ and husbands of centenarians $(\mathrm{p}=0.04)$. On the other hand, mothers of centenarians (who survived up to the age of 50 years) have the shortest life span among all relatives (on average 77.2 years). The relatively low life span of mothers of centenarians is most likely related to the physiological burden of childbearing in these large families, which was shown to increase the mortality of mothers having many children [27]. Overall, siblings-in-law have the lowest life span compared to biological relatives and spouses born in a similar time period. This result is not surprising taking into account that siblings-in-law do not have the genetic or environmental advantages of other relatives of centenarians. Interestingly, the life span of siblings-in-law is still higher than the mean life span of the general population (1900 US birth cohort). This difference is particularly high for men (1.7 years, $p<0.001$ ), while for women it is not statistically significant (table 2). This finding indicates that comparing the survival of siblings or other biological relatives of centenarians to the general population may overstate their survival advantage and hence overestimate the genetic contribution to life span. Thus, a proper control group should be siblings-in law, rather than a general population.

It was found that male gender of centenarians has significant positive effect on the survival of adult male relatives (brothers and fathers), but not of female relatives [24]. A comparison between married siblings and siblings-in-law of centenarians found a strong positive effect of centenarian male gender on the survival of brothers and a weak positive effect of female gender on the survival of centenarian sisters compared to the same-sex siblings-in-law. Figure 1 shows survival curves after the age of 30 years for male siblings of centenarians depending on centenarian gender. Note that brothers of male centenarians have a substantially better survival than brothers of female centenarians, and this difference in survival is highly statistically significant $(\mathrm{p}<0.001)$ according to the generalized Wilcoxon test. This survival advantage is particularly strong after the age of 65 years, whereas the differences in survival before the age of 50 years are minimal. Thus, having a centenarian brother is associated with better late-life survival for males. Taking into account that female gender of centenarians has a much weaker effect on the survival of sisters than male gender of centenarians has on the survival of brothers, 


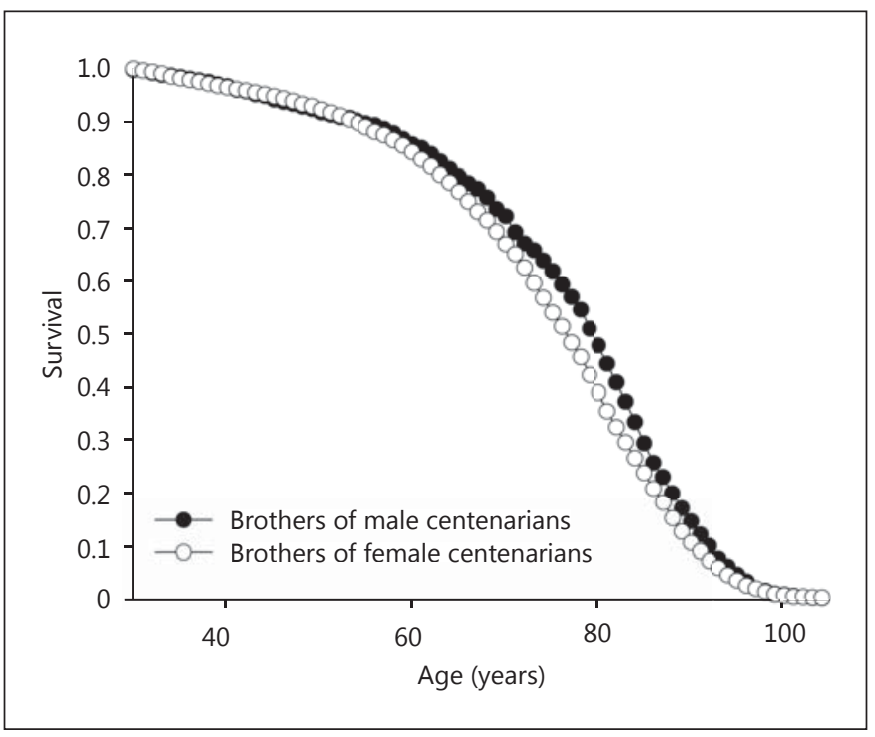

Fig. 1. Survival curves for male siblings (brothers) of centenarians by centenarian gender.

we may hypothesize that male centenarians and their brothers share living conditions and lifestyles favorable for men. This favorable lifestyle could come from the fathers, whose businesses and occupation were often inherited by sons in the past [28]. For example, it has been shown that farming is beneficial for the longevity of males [25]. Thus, it is reasonable to suggest that brothers of male centenarians shared the farming occupation of their fathers and long-lived brothers, which contributed to their survival advantage. On the other hand, female centenarians have the same likelihood to live on a farm or be married to a farmer as shorter-lived female controls [24]. Thus, we may suggest that brothers of female centenarians have the same likelihood to be a farmer as brothers of shorter-lived controls and hence do not have an additional survival advantage related to farming. This explanation is also consistent with our earlier findings as well as results of other studies, which found positive effects of farming and a farm background on late-life survival $[29,30]$. Finally, wives of male centenarians had a significantly better survival compared to wives of brothers of centenarians, indicating the importance of withinfamily living conditions and lifestyles in longevity determination. As expected, centenarian gender had no effect on the survival of centenarian siblings-in-law. This study suggests that intrafamilial environmental conditions and lifestyles may play a more significant role in the exceptional longevity than it was thought before.

New Developments in the Biodemography of Aging and Longevity

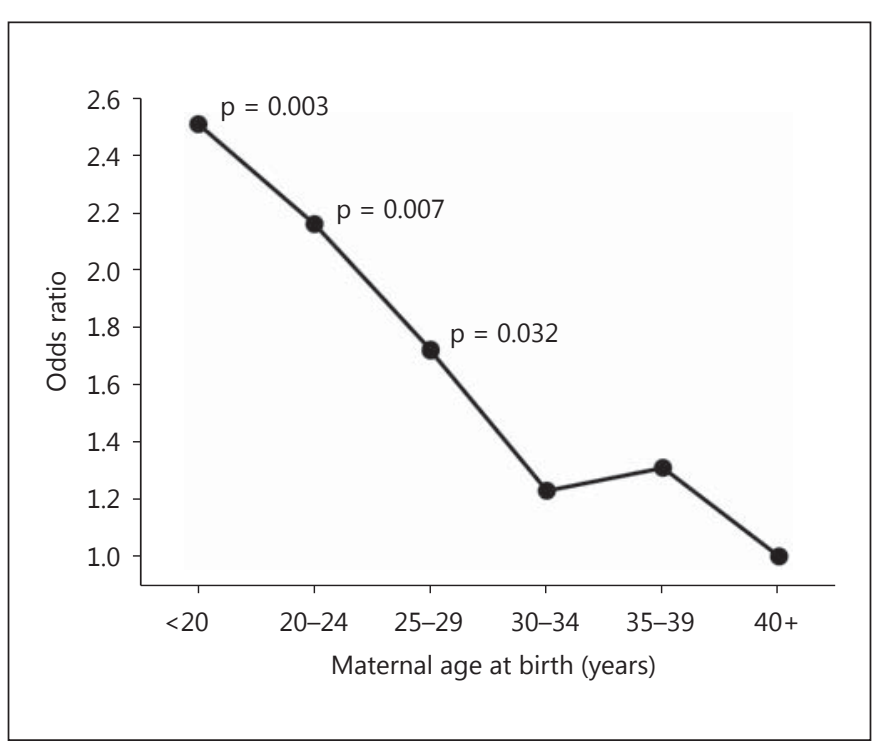

Fig. 2. Effects of maternal age at an individual's birth on the odds to live up to 100 years (conditional on survival up to the age of 50 years). A within-family study of 2,153 centenarians and their siblings who survived up to the age of 50 years using data of smaller families with less than 9 children. Calculated using Stata 11 statistical package (clogit procedure).

\section{Why Centenarians Are Different from Their}

\section{Shorter-Lived Siblings}

Siblings share early-childhood conditions, including parental socioeconomic status, genetic background, and geographical location, while spouses share common adulthood environments. It has been shown that longevity has a significant familial component, suggesting the need to control for this important factor. A comparison of centenarians and their shorter-lived siblings was conducted using a within-family analysis via the method of conditional logistic regression, which allows researchers to control for unobserved shared childhood or adulthood environments and common genetic backgrounds. We have developed and analyzed a computerized database of 1,711 validated centenarians born in the United States between 1880 and 1895 as well as of their shorter-lived siblings [24].

We first explored the effects of parental age at a person's birth on chances of survival up to the age of 100 years and found significant beneficial effects of a young maternal age at a person's birth on survival up to the age of 100 years, with a particularly strong positive influence at a maternal age of 20-24 years [24, 25, 31]. The effect of a young mother was particularly prominent in smaller families [24], which is pertinent today because of the smaller average family size in contemporary population (fig. 2). Thus, the 
within-family analysis of the paternal and maternal age effects on human longevity demonstrated that a young age of the mother increases the chances of children to reach longevity. The finding of a beneficial effect of young maternal age on offspring survival up to the age of 100 years in humans is also reported for laboratory animals [32,33] and hence may have a biological explanation. There is empirical evidence that the quality of female eggs in human beings rapidly declines with age, and this deterioration starts rather early - before the age of 30 years [34].

In addition to more general biological mechanisms, some additional sociobehavioral mechanisms may be involved in human beings. One such mechanism may be the positive effects of prolonged maternal care on health and longevity or the so-called 'mothering hypothesis' [29]. According to this hypothesis, maternal age effects in human beings are mediated through the length of mothering or exposure to the maternal care. Some researchers believe that the observed relationship between maternal age and mortality is most likely explained by the effects of early maternal loss among the children of old mothers rather than physiological mechanisms related to maternal aging [35]. Specifically, it was found that the detrimental effects of old maternal age disappear after controlling for maternal education and life span overlap between the child and the mother. In our study, we used the withinfamily approach, which is more robust to the effects of the parental socioeconomic status and, particularly, of maternal education. Almost all mothers in our sample were housewives and finished their education by the time of marriage. As a result, maternal education remained the same for all the siblings in the family. In addition to that, controlling for maternal longevity did not result in a disappearance of the effects of maternal age on longevity. Although late-born children of long-lived mothers have a substantial exposure to maternal care (exceeding 35 years), they still demonstrate a lower likelihood of survival up to the age of 100 years. Thus, the maternal age effects on survival found in the within-family analysis are more likely to be related to some physiological mechanisms. Similar results were obtained in other independent studies of maternal age effects on longevity applying the within-family approach $[25,29,36]$.

These results provide evidence that early-life programming influences human longevity. A recent study of Canadian centenarians [36], which applied the same withinfamily approach, confirmed our initial finding of an inverse relationship between maternal age and thelikelihood of survival up to the age of 100 years [25]. Another recent study of historical Swedish cohorts [37] also demonstrat- ed beneficial effects of a young maternal age on the life span of offspring.

In addition to maternal age, the within-family approach can be applied to the studies of season-of-birth effects on exceptional longevity. The month of birth is a useful proxy characteristic for seasonal environmental effects acting in utero and during early infancy. Little information is available on the association between the month of birth and exceptional longevity. To analyze net effects of the month of birth on exceptional longevity, not confounded by possible changes in birth and infant death seasonality, childhood conditions, and genetic background, we conducted a matched study using a multivariate conditional logistic regression method [38]. The month of birth of 1,574 validated centenarians born in the United States during 1880-1895 were compared to the same information obtained for 10,885 shorter-lived siblings and 1,083 spouses. This within-family analysis found that the months of birth have significant long-lasting effects on survival up to the age of 100 years; adult siblings born in September-November had significantly higher odds of becoming centenarians compared to siblings born in March. A similar month-of-birth pattern was found for spouses of centenarians. This differential survival leads to an excess of fall-born persons among centenarians [38]. These findings are in a good agreement with our earlier reports on the effects of the month of birth on mortality in the United States [39], and are consistent with our study of centenarians and shorter-lived peers (see above). The results of our study are based on a more conclusive within-family analysis and were not confounded by between-family variation. They demonstrate that the month of birth has a strong independent effect on human longevity. Similar results were obtained using three different data sources. The findings of the within-family analyses support the idea of early-life programming of human aging and longevity.

This study on exceptional longevity investigates the biological and social correlates of why some people survive to extreme old age (older than 100 years). Some results of this study may have practical implications. Specifically, we found beneficial effects of young maternal age at persons' birth on their longevity $[24,25]$. This finding highlights the consequences of delaying childbearing (becoming more common in developed countries) on offspring's health. Studies on links between the season of birth and human longevity $[24,38]$ emphasized important effects of early-life conditions on late-life survival and suggested that environmental conditions and lifestyles may be as important as genetics in determining lon- 
gevity. We also found that mortality (hazard rate) after the age of 100 years does not decelerate but follows the traditional Gompertz law [15, 17]. This finding will allow demographers and gerontologists to improve forecasts of human mortality and the size of the older population. These are all significant issues in the rapidly expanding field of biodemography of aging and longevity.

\section{Acknowledgements}

We would like to acknowledge support from the U.S. National Institute on Aging (NIA grant R01 AG028620).

\section{References}

1 Gavrilov LA, Gavrilova NS: The Biology of Life Span: A Quantitative Approach. New York, Harwood Academic Publisher, 1991.

2 Gavrilov LA, Gavrilova NS: Reliability theory of aging and longevity; in Masoro EJ, Austad SN (eds): Handbook of the Biology of Aging. San Diego, Academic Press, 2006, pp 3-42.

-3 Carnes BA, Olshansky SJ, Grahn D: Continuing the search for a law of mortality. Popul Dev Rev 1996;22:231-264.

4 Olshansky SJ: On the biodemography of aging: a review essay. Popul Dev Rev 1998;24:381393.

5 Greenwood M, Irwin JO: The biostatistics of senility. Hum Biol 1939;11:1-23.

-6 Carey JR, Liedo P, Orozco D, Vaupel JW: Slowing of mortality-rates at older ages in large medfly cohorts. Science 1992;258:457-461.

7 Mueller LD, Rauser CL, Rose MR: Does Aging Stop? Oxford, Oxford University Press, 2011.

8 Beard RE: Note on some mathematical mortality models; in Wolstenholme EW, O'Connor MO (eds): The Lifespan of Animals. Boston, Little, Brown and Co., 1959, pp 302-311.

9 Sacher GA: The Gompertz transformation in the study of the injury-mortality relationship: application to late radiation effects and ageing; in Lindop PJ, Sacher GA (eds): Radiation and Aging. London, Taylor and Francis, 1966, pp 411-441.

10 Austad SN: Concepts and theories of aging; in Masoro EJ, Austad SN (eds): Handbook of the Biology of Aging. San Diego, Academic Press, 2001, pp 3-22.

-11 Bronikowski AM, Alberts SC, Altmann J, Packer C, Carey KD, Tatar M: The aging baboon: comparative demography in a non-human primate. Proc Natl Acad Sci USA 2002;99:95919595.

12 Bronikowski AM, Altmann J, Brockman DK, Cords M, Fedigan LM, Pusey A, Stoinski T, Morris WF, Strier KB, Alberts SC: Aging in the natural world: comparative data reveal similar mortality patterns across primates. Science 2011;331:1325-1328.

13 Horiuchi S, Wilmoth JR: Deceleration in the age pattern of mortality at older ages. Demography 1998;35:391-412.

14 Thatcher AR, Kannisto V, Vaupel JW: The Force of Mortality at Ages 80 to 120. Odense, Odense University Press, 1998.
Gavrilov LA, Gavrilova NS: Mortality measurement at advanced ages: a study of the Social Security Administration Death Master File. N Am Actuar J 2011;15:432-447.

16 Gavrilova NS, Gavrilov LA: Stárnutí a dlouhověkost: zákony a prognózy úmrtnosti pro stárnoucí populace (in Czech). Demografie 2011:53:109-128.

17 Gavrilova NS, Gavrilov LA: Biodemography of old-age mortality in humans and rodents. J Gerontol A Biol Sci Med Sci 2014, DOI: 10.1093/gerona/glu009, Epub ahead of print.

18 Coale AJ, Kisker EE: Mortality crossovers - reality or bad data. Popul Stud 1986;40:389-401.

19 Modig K, Drefahl S, Ahlbom A: Limitless longevity: comment on the contribution of rectangularization to the secular increase of life expectancy. Int J Epidemiol 2013;42:914-916.

20 Robine JM, Vaupel JW: Supercentenarians: slower ageing individuals or senile elderly? Exp Gerontol 2001;36:915-930.

21 Engberg H, Oksuzyan A, Jeune B, Vaupel JW, Christensen K: Centenarians - a useful model for healthy aging? A 29-year follow-up of hospitalizations among 40,000 Danes born in 1905. Aging Cell 2009;8:270-276.

22 Finch CE, Crimmins EM: Inflammatory exposure and historical changes in human lifespans. Science 2004;305:1736-1739.

23 Genetic epidemiologic studies on age-specified traits. NIA Aging and Genetic Epidemiology Working Group. Am J Epidemiol 2000;152: 1003-1008.

24 Gavrilov LA, Gavrilova NS: Determinants of exceptional human longevity: new ideas and findings. Vienna Yearb Popul Res 2013;11: 295-323.

25 Gavrilov LA, Gavrilova NS: Biodemography of exceptional longevity: early-life and mid-life predictors of human longevity. Biodemography Soc Biol 2012;58:14-39.

26 Travers C, Bartlett HP: Silver memories: implementation and evaluation of a unique radio program for older people. Aging Ment Health 2011;15:169-177.

27 Gagnon A, Smith KR, Tremblay M, Vezina H, Pare PP, Desjardins B: Is there a trade-off between fertility and longevity? A comparative study of women from three large historical databases accounting for mortality selection. Am J Hum Biol 2009;21:533-540.
28 Ruggles S: The decline of intergenerational coresidence in the United States, 1850 to 2000. Am Sociol Rev 2007;72:964-989.

29 Gavrilova NS, Gavrilov LA: Search for predictors of exceptional human longevity: using computerized genealogies and internet resources for human longevity studies. N Am Actuar J 2007;11:49-67.

30 Preston SH, Hill ME, Drevenstedt GL: Childhood conditions that predict survival to advanced ages among African-Americans. Soc Sci Med 1998;47:1231-1246.

31 Gavrilova NS, Gavrilov LA: Search for mechanisms of exceptional human longevity. Rejuvenation Res 2010;13:262-264.

- 32 Tarin JJ, Gomez-Piquer V, Rausell F, Navarro S, Hermenegildo C, Cano A: Delayed motherhood decreases life expectancy of mouse offspring. Biol Reprod 2005;72:1336-1343.

33 Carnes BA, Riesch R, Schlupp I: The delayed impact of parental age on offspring mortality in mice. J Gerontol A Biol Sci Med Sci 2012; 67:351-357.

34 Heffner LJ: Advanced maternal age - how old is too old? N Engl J Med 2004;351:1927-1929.

- 35 Myrskyla M, Fenelon A: Maternal age and offspring adult health: evidence from the health and retirement study. Demography 2012;49: 1231-1257.

-36 Jarry V, Gagnon A, Bourbeau R: Maternal age, birth order and other early-life factors: a family-level approach to exploring exceptional survival. Vienna Yearb Popul Res 2013;11: 263-284.

37 Wilding M, Coppola G, De Icco F, Arenare L, Di Matteo L, Dale B: Maternal non-Mendelian inheritance of a reduced lifespan? A hypothesis. J Assist Reprod Genet 2014;31:637643.

38 Gavrilov LA, Gavrilova NS: Season of birth and exceptional longevity: comparative study of American centenarians, their siblings, and spouses. J Aging Res 2011;2011:104616.

39 Gavrilov LA, Gavrilova NS: Mortality Measurement at Advanced Ages: A Study of the Social Security Administration Death Master File - Living to 100 and Beyond: Survival at Advanced Ages (online monograph). Shaumburg, The Society of Actuaries, 2008, 32 pages. 